\title{
Failure mechanisms of polyimide and perfluoroalkoxy films under high frequency pulses
}

\author{
Weijun Yin ${ }^{1}$, Fengfeng Tao ${ }^{1}$, Junwei Zhao ${ }^{2}$, George Chen $^{2}$ and Daniel Schweickart ${ }^{3}$ \\ ${ }^{1}$ GE Global Research Center, One research Circle, Niskayuna, NY12309, USA \\ ${ }^{2}$ University of Southhampton, Southampton, SO17 1BJ, UK \\ ${ }^{3}$ Air Force Research Laboratory, Wright Patterson Air Force Base, OH, USA
}

\begin{abstract}
Breakdown behaviors of polyimide and perfluoroalkoxy high temperature films under unipolar and bipolar repetitive pulses are investigated. A bipolar $20 \mathrm{kV}, 20 \mathrm{kHz}$ pulse generator with fast $\mathrm{dV} / \mathrm{dt}$ pulse risetime has been designed and built to study the impact of pulse frequency, pulse rise time, and pulse polarity and pulse duty cycles on breakdown strength of these films films. Space charge injection and decay processes are also investigated. Possible failure mechanisms are discussed.
\end{abstract}

\section{INTRODUCTION}

Combined stresses from high frequency, high voltage and high temperature at low pressure pose serious problems to insulation life and the full system's reliability. The breakdown behavior of insulation under these environments is not fully understood. Up to now, magnet wire, used in fractional horsepower motors, has been the subject of a good deal of experimentation as a result of the concern for aging caused by adjustable speed drives (ASD). For example, Guastavino et $\mathrm{al}^{1}$ have shown that the number of aging cycles to breakdown for twisted pairs of magnet wire is independent of frequency from $50 \mathrm{~Hz}$ to $5 \mathrm{kHz}$. Cerutti et $\mathrm{al}^{2}$ showed similar data from 1.5 to $5 \mathrm{kHz}$. Yin ${ }^{3}$, however, not only showed similar data from $200 \mathrm{~Hz}$ to $5 \mathrm{kHz}$, but also discovered that the number of aging cycles to breakdown decreases with increasing frequency when frequency is beyond $5 \mathrm{kHz}$. All these tests mentioned were conducted in air where surface corona had an effect on breakdown.

It is attempted in this paper to address the concern for sustainability and reliability of electrical insulation, especially polymeric dielectric materials to be used in compact and lightweight power systems in extreme environments. For example, insulation system used at operating conditions at temperatures greater than $250^{\circ} \mathrm{C}$ and under electrical stresses arising from high frequency power converters with high $\mathrm{dV} / \mathrm{dt}$ switching. Our goal is to understand high frequency failure mechanisms in current commercial insulating materials operating at extremely high temperatures. This data can provide the technology baseline necessary to define the development of new robust high temperature and high frequency insulation through the application of nanotechnology.

Commercial-off-the-shelf (COTS) high frequency high voltage power supplies did not meet our testing requirement. They either lack output voltage level, frequency or risetime. Some U.S. National labs, such as Sandia National Labs, have specially designed and built pulse generators, but they are not commercially available. With this program, we have successfully designed and built a bipolar $20 \mathrm{kV}, 20$ $\mathrm{kHz}$ pulse generator with fast $\mathrm{dV} / \mathrm{dt}(20 \mathrm{kV} / \mu \mathrm{s})$ rise time to meet voltage amplitude, pulse rise time, frequency range, and capacitive load requirements.
The impact of transient voltages with fast switching frequency on current state of art high temperature insulation materials, such as polyimide (PI), and perfluoroalkoxy (PFA) films are investigated here. Effects of pulse frequency, pulse rise time, and pulse polarity on breakdown behaviors of these films are determined. Space charge accumulation and relaxation behaviors are also investigated. Failure mechanisms are discussed and compared to that for DC and power frequency AC breakdown.

\section{EXPERIMENTAL}

\section{A. Materials}

Five different types of high temperature polymeric insulation films were selected to study. They are various polyimide films (conventional-A, corona resistant-B, thermally conductive-C, high heat resistant-D) made by DuPont and UBE; as well as Perfluoroalkoxy (PFA-E) film. All films have thickness of 25 microns. These solution-cast insulations typically have application as insulation for magnet wire in random-wound electrical machines and magnetic devices.

\section{B. Breakdown tests}

Hipotronics $\mathrm{AC} / \mathrm{DC}$ breakdown tester was used to measure $\mathrm{AC}$ and DC breakdown voltages of films before and after thermal aging. A $1 / 4$ " stainless steel ball was used as HV electrode and a smooth ferro plate was used as ground electrode. Films were immersed in silicone oil for breakdown tests. A $500 \mathrm{~V} / \mathrm{sec}$ ramp rate was selected for measurement.

A custom-built $20 \mathrm{kHz} \mathrm{HV}$ pulse generator was employed to measure the film breakdown voltages under both unipolar and bipolar pulses. The test set-up for these experiments is shown in Figure 1. The samples were subjected to $20 \mathrm{kHz}$ pulsed voltages with $\mathrm{dV} / \mathrm{dt}$ of $20 \mathrm{kV} /$ microsec. The ramp rate used for increasing voltage is about $410 \mathrm{~V} / \mathrm{sec}$ (slightly slower than $500 \mathrm{~V} / \mathrm{sec}$ used for both DC and $60 \mathrm{~Hz}$ $\mathrm{AC}$ sine wave). The actual breakdown value of the film is recorded when the sample fails.

All films used for pulse breakdown tests were preconditioned in dry desiccators for 48 hours before test. Fresh silicone oil was used as the testing medium for sample immersion, to prevent surface flashover. All films have 1 mil in thickness, so breakdown voltage can be used for direct comparison. A $1 / 2$ " stainless steel Rogowski electrode was used as the positive HV electrode and a smooth ferroplate was used as the ground or negative HV electrode. A Teflon 
block was used to stabilize the top electrode. The stem of the top electrode was centered in the middle of the Teflon block. Good contact of electrode to the film was maintained by the own weight of the top electrode (about 20g), as shown in Figure 2. Films are tested under both bipolar and unipolar pulse conditions to see if polarity plays a role in breakdown. Typical pulse waves used for testing are shown in Figure 3 and 4. Figure 3 shows pulse waveforms of $20 \mathrm{kHz}$ bipolar pulse with $50 \%$ duty cycle and Figure 4 shows pulse waveforms of $20 \mathrm{kHz}$ bipolar pulse with $10 \%$ duty cycle. The ramp rate to increase voltage is controlled manually to about $\sim 410 \mathrm{~V} / \mathrm{sec}$, as shown in Figure 5. At least 10 breakdown points were measured for each pulse condition for each sample.

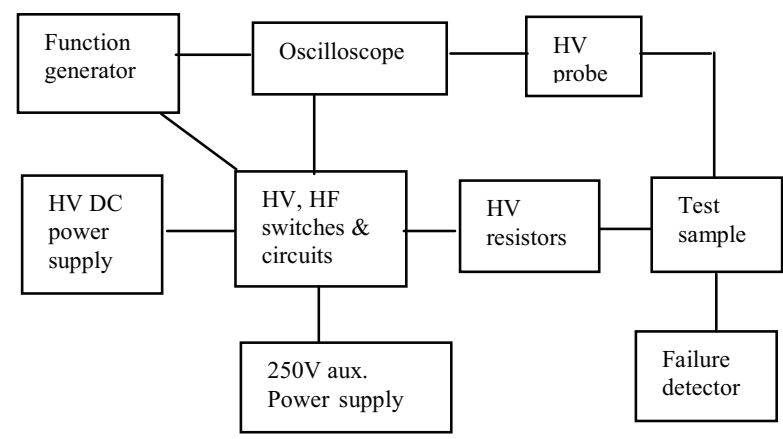

Fig.1 Pulse test schematic set-up

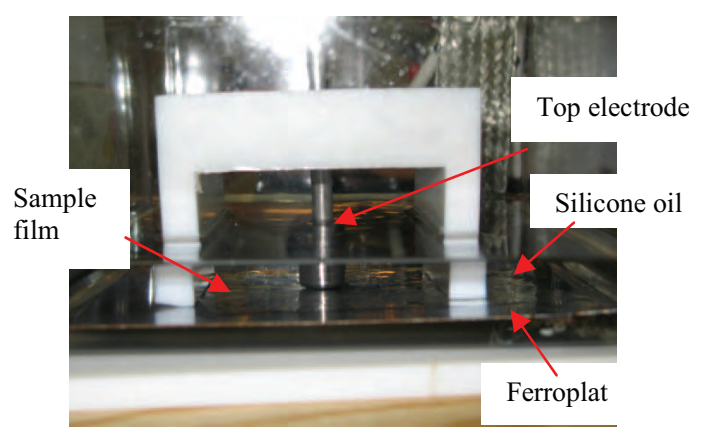

Fig.2 Picture of sample set up for pulse breakdown test

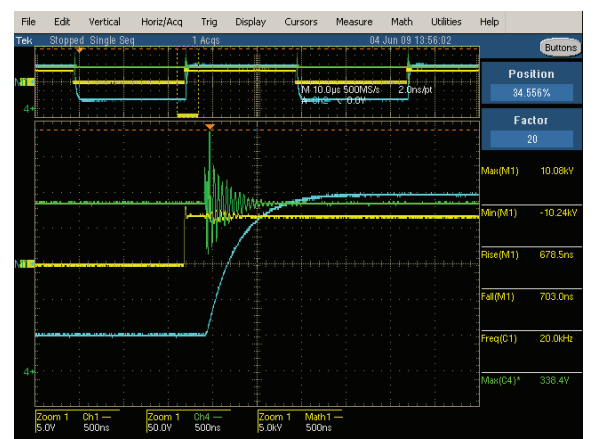

Fig. $320 \mathrm{kHz}, 50 \%$ duty bipolar pulse waveform applied on sample

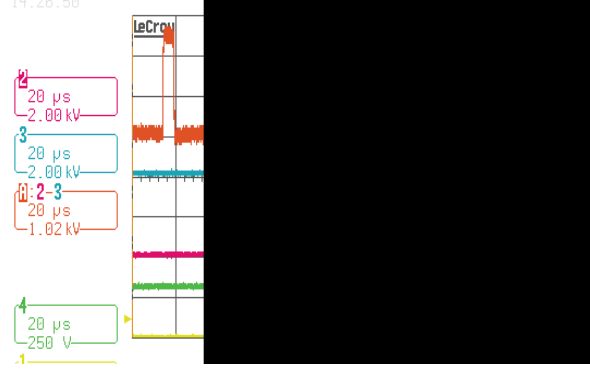

Fig.4 $20 \mathrm{kHz}, 10 \%$ duty bipolar pulse waveform applied on sample

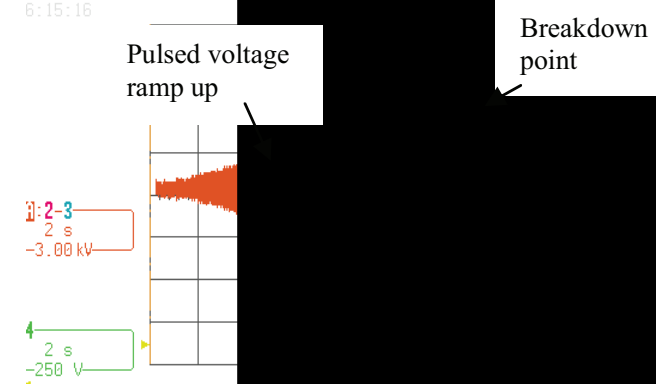

Fig.5 Ramp up voltage waveform for bipolar pulse breakdown

\section{Space charge measurement}

The pulsed electro-acoustic (PEA) method was used to measure the dynamics of space charge within films under dc voltages due to its quick response and high spatial resolution. The schematic of PEA technique is illustrated in figure 6 . DC bias voltage was applied across the sample film mounted between the top and ground electrodes. As soon as the pulse voltage was applied, the detected electrical signal was amplified and captured by a digital oscilloscope (DSO) and transferred to a computer program for deconvolution. The spatial resolution of this technique was about $5 \mu \mathrm{m}$ due to the narrow pulse voltage width of $2 \mathrm{~ns}$.

Film samples were cut into small discs with a diameter of $\Phi=28 \mathrm{~mm}$ and measured using the PEA kit. All samples were first polarized under $1 \mathrm{kV}$ dc voltage at the ambient temperature for one hour, the PEA output signals were recorded after one hour polarization followed by an immediate depolarization process for a second hour where the sample was short-circuited.

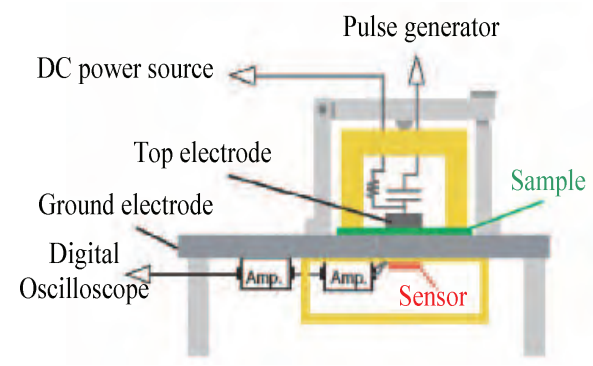

Fig.6 Schematic of PEA set up 


\section{RESULTS AND DISCUSSIONS}

\section{A. Bipolar pulses}

Breakdowns of films under high frequency pulses are pin hole failures. The failure spot is very small compared to sinusoidal $60 \mathrm{~Hz}$ $\mathrm{AC}$ breakdown. The pinhole size is less than 500 micron.

Since the ranges of breakdown voltages for all PI films are overlapping, it is hard to show results in Weibull plots. Box plots (shown in Figure 7) are used to display breakdown voltages for various films under bipolar square wave pulses at $20 \mathrm{kHz}$ and $50 \%$ duty cycles with a rise and fall rate about $20 \mathrm{kV} / \mathrm{us}$. The box shows $75 \%$ of data points, and the red line is the indication of median value. Quite a wide range for breakdown values noticed for sample D. All polyimide films (A-D) have similar breakdown values under bipolar pulses at about $7 \mathrm{kV}$ peak to peak, while PFA film has higher breakdown value about $9 \mathrm{kV}$ peak-to-peak, where red line is the median value of 10 breakdown points on each sample. All polyimide films have breakdown values in the close range having overlaps, so they are not significantly different. However, PFA film shows significantly higher values than all PI films. Since modulus of PI films (2500MPa for sample A) are significantly higher than that of PFA film (480MPa) ${ }^{4}$, while the ratio difference in dielectric constant is less than 2 (3.4 for sample A and 2.0 for sample E) it is apparent that electro-mechanical failure is not the failure mechanism for high frequency pulse breakdown. Because the electro-mechanical breakdown field strength $\left(\mathrm{E}_{\mathrm{m}}\right)$ can be expressed as $\mathrm{E}_{\mathrm{m}}=\mathrm{A}(\mathrm{Y} / \varepsilon)^{1 / 2}$ (where $\mathrm{A}$ is a constant, $\mathrm{Y}$ is the Young's modulus and $\varepsilon$ is the dielectric constant ${ }^{5}$ ), the calculated $\mathrm{E}_{\mathrm{m}}$ of sample $\mathrm{A}$ is three times of $E_{m}$ of sample $E$, which is opposite of what was measured experimentally.

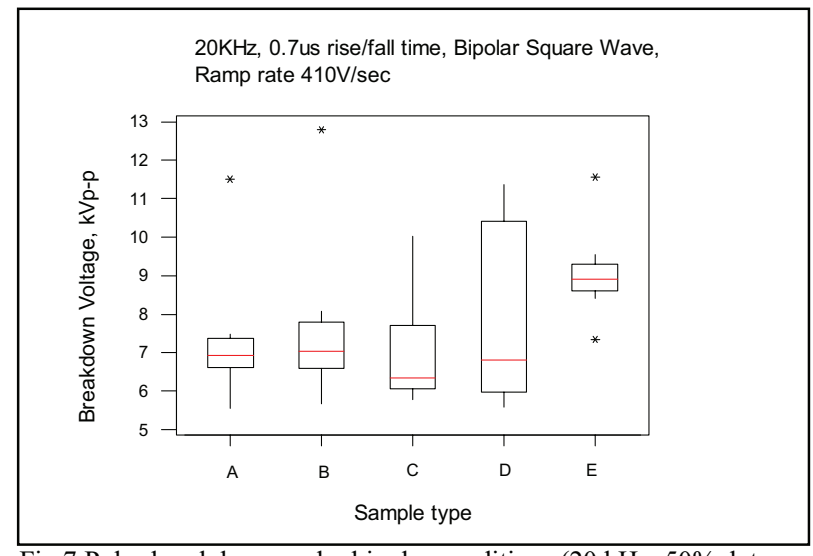

Fig.7 Pulse breakdown under bipolar conditions $(20 \mathrm{kHz}, 50 \%$ duty, and $20 \mathrm{kV} /$ microsecond rise and fall)

\section{Frequency effect:}

Pulse frequency effect on breakdown strength of these films was investigated to determine if electro-thermal failure could be one of the failure mechanisms. Regular PI film (sample A) thermally conductive PI film (sample C) and low dielectric loss PFA film (sample E) were selected for comparison.

Figure 8 shows frequency dependency of breakdown voltages for various films. All films have the same thickness of 1 mil. Each point in the curve is the alpha value of Weibull plot of 10 test points for each sample at each condition. At $20 \mathrm{kHz}, \mathrm{PI}-\mathrm{A}$ and PI-C have similar breakdown values, however, the breakdown value for PI-A increases significantly with decreasing frequency while breakdown values of PI-C does not change much with decreasing frequency. In other words, PI-C is less sensitive to pulse frequency. This could be explained by electro-thermal failure mechanism. The energy generated in the film during voltage ramp up is cumulative, which should be proportional to the number of cycles (the product of pulse frequency and time to failure). At low pulse frequency, the heat is easily dissipated and breakdown value is more or less defect controlled for films with the same thickness. Clearly, PI-C is less uniform in structure than PI-A due to addition of micro sized alumina. More defects in PI-C can be expected, therefore lower breakdown strength. However, with increasing pulse frequency, if the rate of heat dissipation is less than heat accumulation, thermal runaway can happen and result in early breakdown. Since the thermal conductivity of PI-C $(0.37 \mathrm{~W} / \mathrm{m} . \mathrm{K})$ is three times higher than that of $\mathrm{PI}-\mathrm{A}(0.12 \mathrm{~W} / \mathrm{m} . \mathrm{K})^{4}$, the heat can be dissipated faster for $\mathrm{C}$ film than A film, therefore, thermal runaway is delayed for sample $\mathrm{C}$ at high pulse frequency. That could be why PI-C does not show as significant drop in breakdown value as does sample A at $20 \mathrm{kHz}$

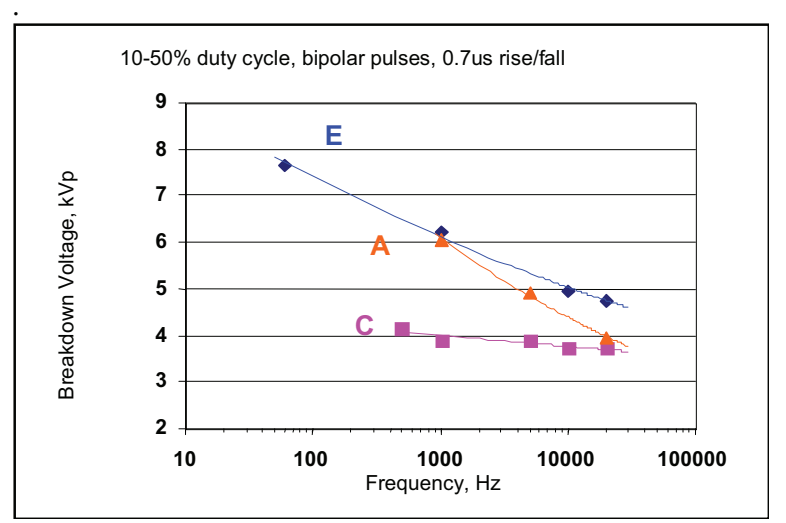

Fig. 8 Breakdown voltage vs. pulse frequency under bipolar pulses for various films

Comparing PFA and PI, although PFA (sample E) also shows increasing breakdown value with decreasing frequency, the slope of change for $\mathrm{E}$ is less than $\mathrm{A}$. The reason for that could be explained by the difference in dielectric dissipation factor or dielectric loss. The PFA film has much lower dielectric loss than PI film, 0.0002 for PFA at $100 \mathrm{~Hz}$ to $1 \mathrm{MHz}$ and 0.0018 for PI at $1 \mathrm{kHz}^{4}$, the dielectric heating effect in PFA film can be expected to be less than PI. The slope difference in frequency dependency may well be explained by the difference in dielectric heating and dissipation for different materials.

\section{B. Unipolar pulses}

\section{Polarity effect:}

Figure 9 shows box plots of breakdown values under positive unipolar pulses with the same pulse parameters. The continuous ramp rate is also about $410 \mathrm{~V} / \mathrm{sec}$. However, under unipolar conditions, PID shows lower value than the rest of the polyimide films. Under both bipolar and unipolar conditions, fluoropolymer outperforms polyimide films. The peak-to-peak breakdown voltage of bipolar pulses is similar to peak voltage of unipolar pulses at same pulse frequency. The polarity effect is not significant. 


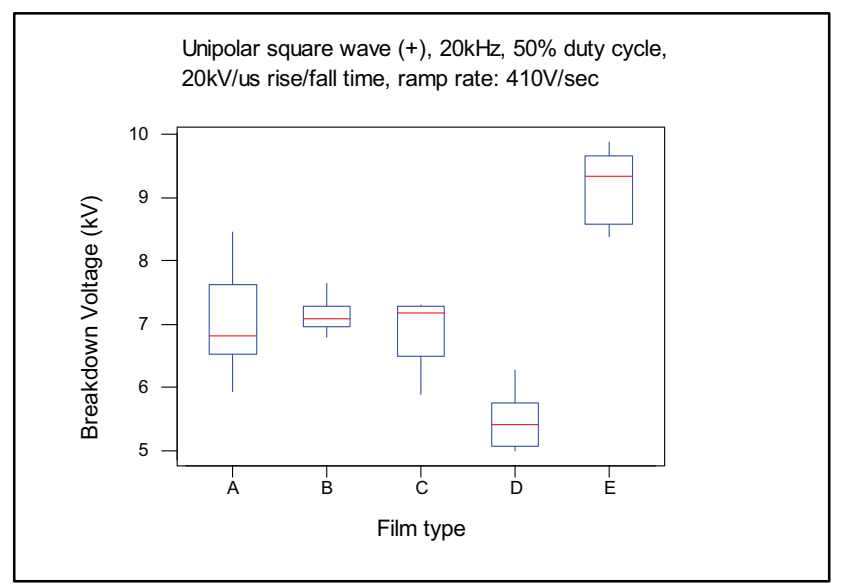

Fig.9 Pulse breakdown under positive unipolar conditions

\section{Duty cycle effects on space charge accumulation:}

To determine if charge accumulation has any effect on the breakdown value under pulse conditions, duty cycles were varied to test film breakdown. If space charge exists, with low duty cycle under unipolar pulse conditions, fewer charges would be injected and charges might have longer time to dissipate, which should result in higher breakdown values as compared to high duty cycle. Figure 10 shows breakdown values under various duty cycles under $20 \mathrm{kHz}$ pulses (the alpha mean value from Weibull distribution of 10 test points was used for each point in the curve), which validates the hypothesis. This space charge effect also contributes to the frequency dependency. At higher pulse frequency, charges accumulate before they can be dispersed or neutralized. The local field can be enhanced by space charges and lead to lower breakdown voltages.

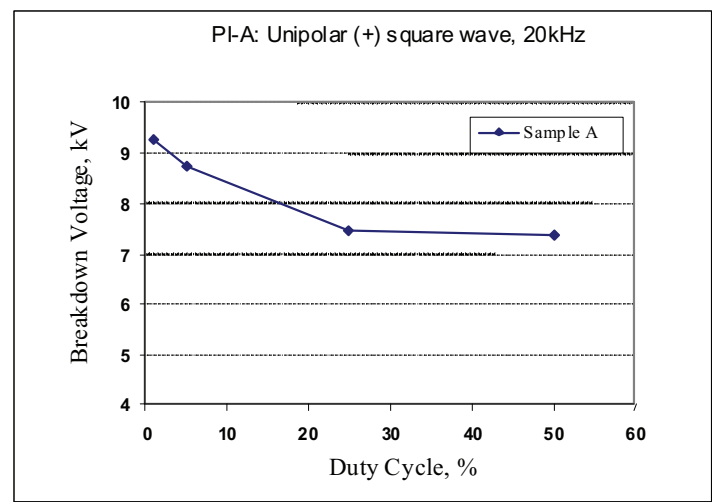

Fig.10 Breakdown voltages of sample A polyimide film under positive unipolar pulses with $20 \mathrm{kHz}, 20 \mathrm{kV} / \mathrm{us}$ rise and fall square wave

\section{Pulse frequency effect:}

Pulse frequency effects on breakdown values were also studied under positive unipolar conditions. Figure 11 shows the results. Note that due to the limitation of $10 \mathrm{kV}$ peak voltage provided by the HV power supply, only one frequency of $20 \mathrm{kHz}$ was used to test sample E under unipolar condition. The breakdown value decreases with increasing frequency for films under both unipolar and bipolar pulses. The frequency dependency of PI-A and PI-B are similar. As was observed under bipolar pulse tests, PI-C shows smaller frequency dependency of breakdown voltage. As discussed in the previous section, the electro-thermal breakdown also accounts for the film failure under high frequency unipolar pulses. By comparing the peakto-peak breakdown voltages under bipolar pulses to the peak breakdown voltages under unipolar pulses (at the same frequency), it seems that polarity change under the bipolar pulse condition does not contribute significantly to the failures.

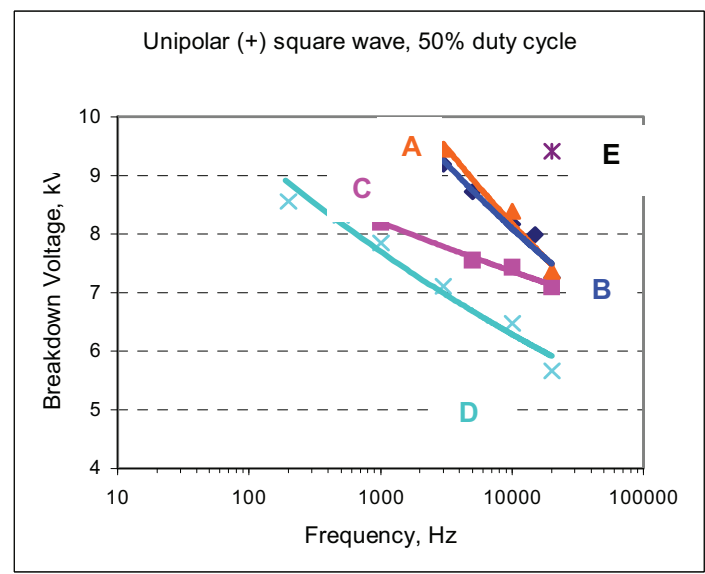

Fig.11 Breakdown voltage vs. pulse frequency under unipolar condition for various films

\section{Space charge}

To understand if space charge plays a role in the pulsed voltage breakdown, we used the pulsed electro-acoustic (PEA) method to investigate the difference in space charge accumulation and relaxation behaviors in all four polyimide films (A to D) and PFA film under DC voltages. A perturbation of the pulse voltage on the internal charges along with those on the electrodes produces acoustic waves that are proportional to the amount of charges and travel through the sample. Such an acoustic signal can be detected by a piezoelectric transducer attached to the ground electrode which converts it into electrical signals. They are then processed into the charge profile by a deconvolution method. Figure $12-21$ show space charge profiles during polarization and depolarization stages for various films.

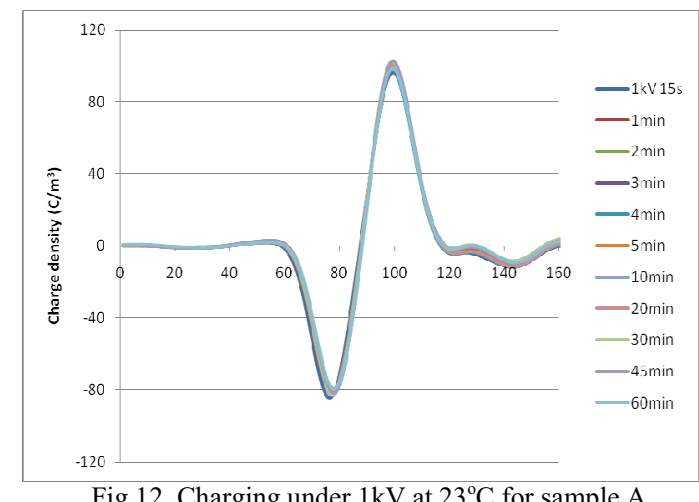

Charge injection takes place from both electrodes for all smaples and mostly symmetrical except sample $E$ which shows larger negative charge injection. The amount of charge injection is not too large for sample A and its charge decay rate is reasonably slow. The amount of charge injection in sample B is very high and its charge decay rate is fast even though its testing temperature is lower. The 


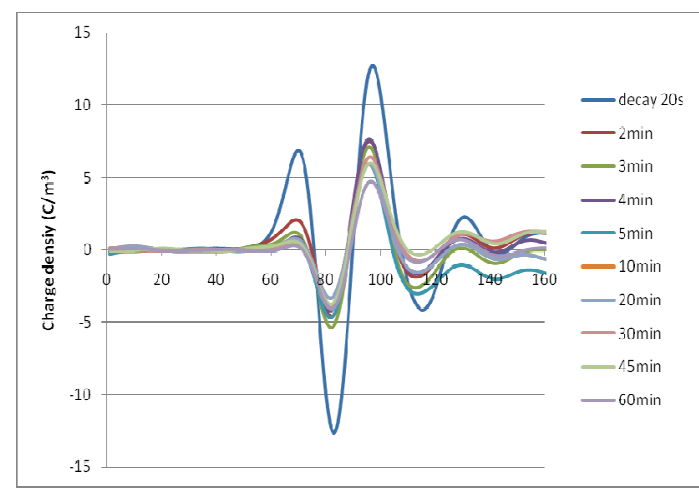

Fig.13 Space charge decaying after short circuited for sample A at $23^{\circ} \mathrm{C}$

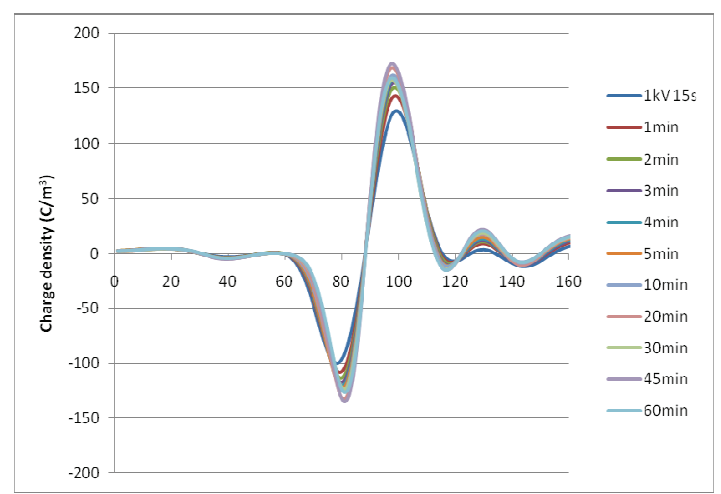

Fig.14 Charging under $1 \mathrm{kV}$ at $14^{\circ} \mathrm{C}$ for sample $\mathrm{B}$

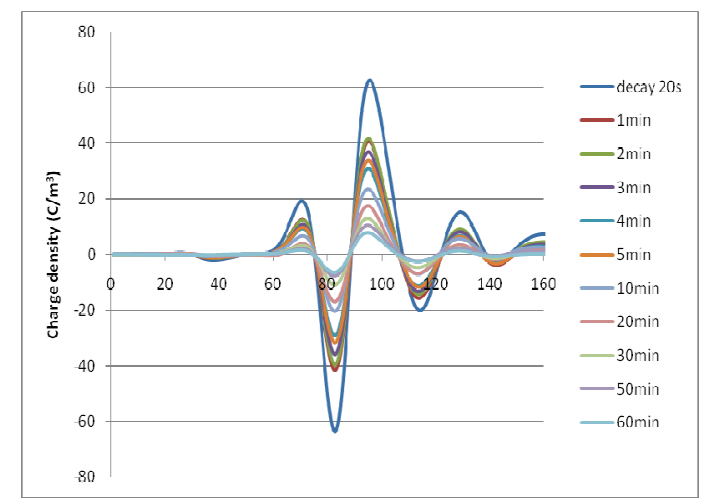

Fig.15 Space charge decaying after short circuited for sample B at $14^{\circ} \mathrm{C}$

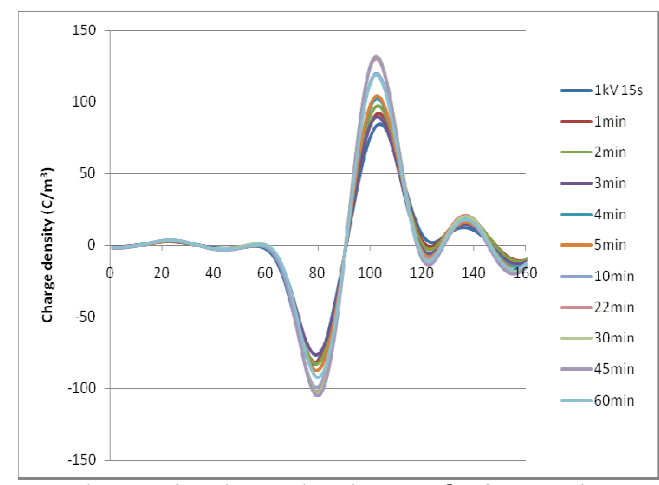

Fig.16 Charging under $1 \mathrm{kV}$ at $25^{\circ} \mathrm{C}$ for sample $\mathrm{C}$

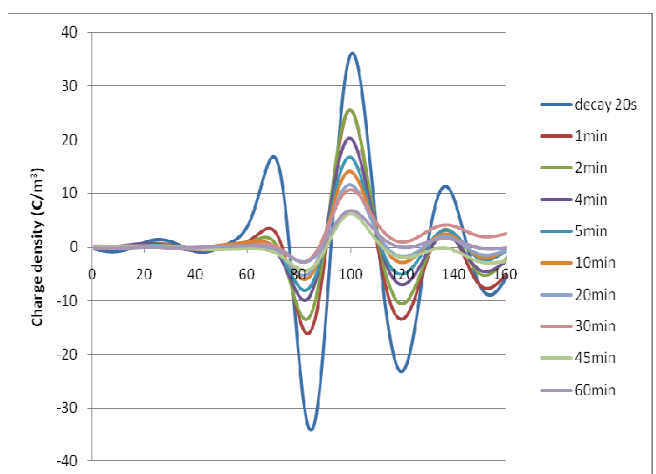

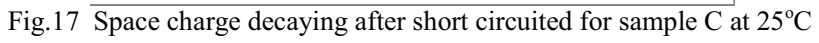

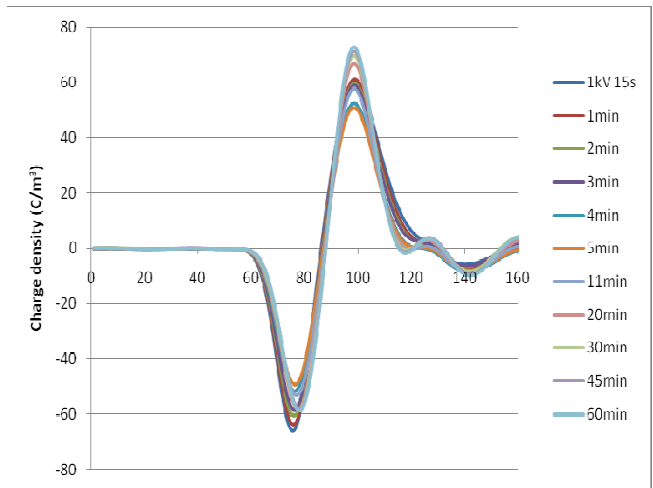

Fig. 18 Charging under $1 \mathrm{kV}$ at $24.5^{\circ} \mathrm{C}$ for sample D

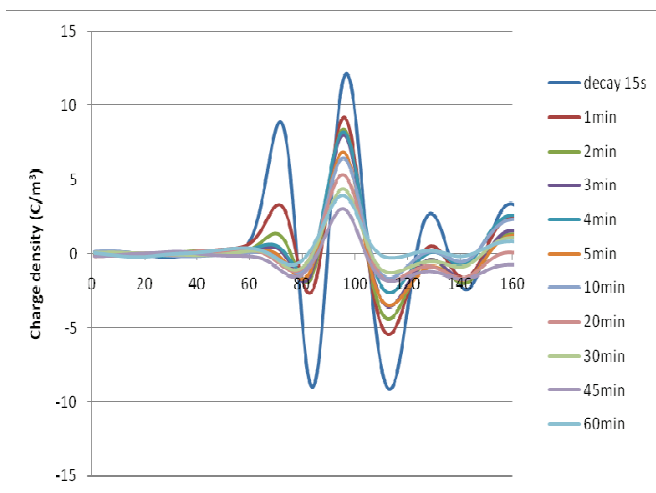

Fig.19 Space charge decaying after short circuited for sample D at $24.5^{\circ} \mathrm{C}$

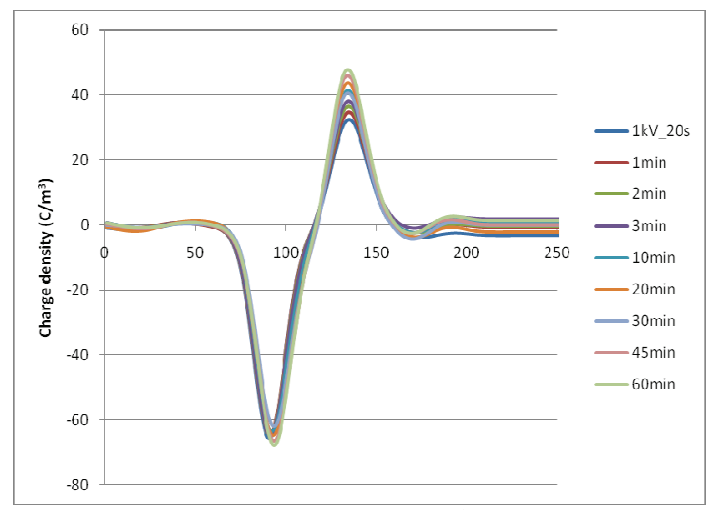

Fig.20 Charging under $1 \mathrm{kV}$ at $14^{\circ} \mathrm{C}$ for sample E 


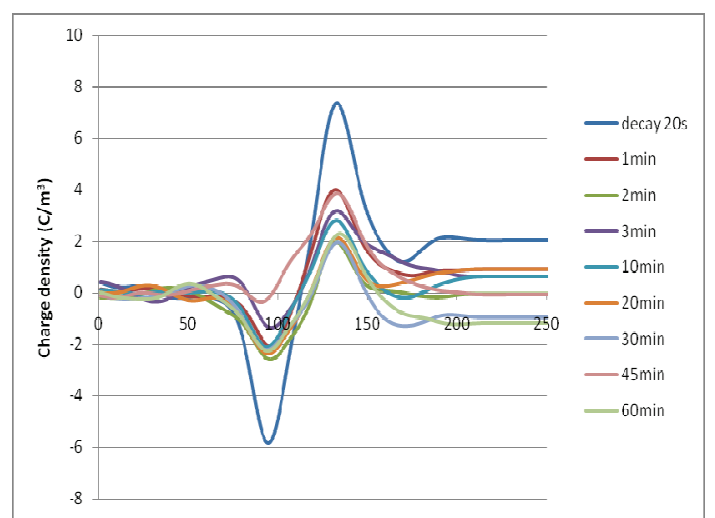

Fig.21 Space charge decaying after short circuited for sample E at $14^{\circ} \mathrm{C}$

amount of charge injection in sample $\mathrm{C}$ is also significant (similar to sample B); its charge decay rate is fast. The amount of charge injection in sample D is not too significant, however, unlike sample A, its charge decay rate is fast. Negative charge is dominating in sample E, however, the amount of charge injection is not significant and its charge decay rate is slow.

At the same stress level, injected charge amounts should be inversly propotional to energy barreier of the material. The larger amount the injected charges, the smaller the band gap of the material, meaning that the material is less insulating. So sample B and C should have lower breakdown strength than sample A, D and E. Sample E should show the highest breakdown strength, which is in consistent with what we observed in DC and pulsed breakdown tests as shown in figure 7 and figure 9 and figure 22. The charge decay rate depends on the trap depth and charge combination sites in the materials. The shallower the trap depth is, the faster the decay rate will be. It is hard to judge insulating properties of materials purely based on decay rate. In short term breakdown tests, the breakdown strength of the material with fast decay rate could be lower due to fast charge mobility. However, in long term endurence tests, charges build up or accumulation could be limited. Local stress build up due to space charge accumulation could be less. In other words, short term breakdown strength of insulation films might be influenced more by charge injection than by charge decay rate. While in long term endurance test, the relationship could flip. Space charge injection under repetitive pulses could be lower compared to DC conditions, due to short pulse duration. However, with increasing pulse frequency and shorter dead time between pulses, charges may accumulate due to insufficient time for charges to be dissipated

\section{Comparison of breakdown strength under AC, DC and Pulsed voltages}

Figure 22 shows comparison results of all five samples tested under sinusoidal AC (60 Hz), DC and pulsed voltages of $20 \mathrm{kHz}$. Mean values from Weibull plots of breakdown results were used in figure 22. The detailed test results of AC and DC breakdown can be found in a previous paper ${ }^{6}$. There is similarity in trend between DC breakdown and pulse breakdown value for various films, which indicates that in addition to electro-thermal stress-induced thermal runaway failure under high frequency pulses, space charge limited current could play a role in dielectric breakdown under repetitive pulses. The reason for lower breakdown values for sample D under unipolar pulses is unclear, but it might be due to defects.

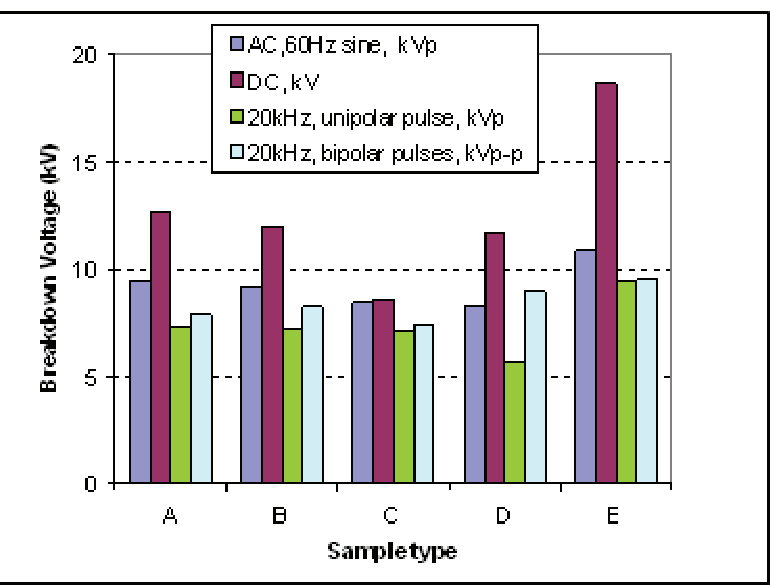

Fig.22 Comparison of breakdown strength of various films under sinusoidal $\mathrm{AC}, \mathrm{DC}$ and $20 \mathrm{kHz}$ pulses

\section{CONCLUSIONS}

Among all the high temperature films tested, PFA films show the highest breakdown strength when tested at room temperature under DC, sinusoidal AC $(60 \mathrm{~Hz})$ and high frequency pulsed voltages at both bipolar and unipolar conditions. The dependence of pulse breakdown on pulse frequency and duty cycle reveals that electrothermal and space charge accumulation are possibly two main failure mechanisms for polymeric material breakdown under highly repetitive pulses. High thermal conductivity and low dielectric loss may retard high frequency pulse breakdown. Space charge measurement results indicate that space charge limited current could play a role in dielectric breakdown in both DC and repetitive high frequency pulses.

\section{ACKNOWLEDGMENT}

Part of work presented in this paper was supported by the Air Force Research Laboratory under the contract of F33615-03-D2352/0010-06. The authors (Yin, Tao) would like to express their gratitude to the financial support from AFRL and thank Mr. Frank Mueller for building high voltage pulse tester.

\section{REFERENCES}

1 F. Guastavino, G. Coletti, and E. Torello. "Medium Term Aging Characterization of Enameled Wires for High Frequency Application". IEEE Trans DEI, Vol. 12, No. 3, June 2005. pp. 524-529.

${ }^{2}$ B. Cerutti., F. Fuastavino, A. Castagnini, and C. Gemme. "Characterization of Insulated Conductors for High Frequency Applications". IEEE 1999 CEIDP, pp. 573-576.

${ }^{3} \mathrm{~W}$. Yin, "Failure mechanism of winding insulations in inverter-fed motors" IEEE Electrical Insulation Magazine, pp.18-23, Vol. 13, No. 6, 1997

${ }^{4}$ DuPont PI and PFA film product literature: http://www.dupont.com

${ }^{5}$ L. A. Dissado and J.C. Fothergill, "Electrical degradation and breakdown in polymers" IEE materials and devices series 9 .

${ }^{6}$ W. Yin and D. Schweickart, "Dielectric Breakdown of Polymeric Insulation Films Under AC, DC and Pulsed Voltages", IEEE EIC, 2009 